\title{
Congenital left lobe megaly of liver resembling splenic pathology: a diagnostic dilemma confirmed bySPECT-CT of liver-spleen scan
}

Puja Bhattacharjee1 ,Tapati Mandal'1, Sharmin Quddus', Pupree Mutsuddi, Papia Akhter, Md. Azharul Islam, Md. Ismail, Abu Bakker Siddique, Reajul Islam, Jasmine Ara Haque

National Institute of Nuclear Medicine and Allied Sciences (NINMAS), Dhaka, Bangladesh

Correspondence Address: : Dr.Puja Bhattacharjee, Medical Officer, Scintigraphy Division, NINMAS, Block-D, BSMMU campus, haka-1000, Bangladesh. E-mail: pujadmc68@gmail.com

\begin{abstract}
Liver scintigraphy is a specialized nuclear medicine imaging technique used to diagnose hepatic pathological conditions as well as to assess the function of the liver. Itis also used to follow the progress of treatment of certain diseases. This procedure may be referred as liver-spleen scan because the spleen is alsoevaluateddue to its proximity and close functional relationship to liver.SPECT-CT correlation along with planar images increases the specificity of the test and can guide the physicians to rule outdiagnostic dilemma regarding certain hepatic \& splenic diseases. Here, we present a case where the patient was initially suspected tohave splenomegaly with splenic hematomabut finally confirmedas unusuallyenlarged left lobe of liverand normal sized spleen with SPECT-CT of liver-spleen scan.
\end{abstract}

Key words: Hepatomegaly, Congenital splenomegaly, Liver-spleen scan. Bangladesh J. Nucl. Med. Vol. 22 No. 2 July 2019

Doi: https://doi.org/10.3329/bjnm.v22i2.51772

\section{INTRODUCTION}

Liver-spleen scintigraphy is a Nuclear Medicine (NM) procedure where a small amount of a radioactive substance is used to assessthe liver. The radiotracer named 99m-Tc Sn-colloid is injected intravenous that travels through the blood to the liver and spleen. It is further detected gamma camera, which forms an image of the areas where the radioactive substance collects.Sn-colloid is comprised of small particles that are phagocytosed by the reticulo-endothelial systems, including Kupffer cells in the liver. Lesions that lack Kupffer cells in the liver will not accumulate the tracer $(10,11)$. This scan may be done for the diagnosis of certain clinical conditions such as- hepatic tumors, hepatitis, cirrhosis, abscesses, cysts of the liver \& spleen. It may also be done to assess the condition of the liver or spleen after abdominal injury or when there is unexplained pain in the right upper quadrant of the abdomen. Enlargement of the liver or spleen and colloid shift may also be noted on this scan (11). When abdominalultrasound finding is inconclusive, liver-spleen scan along with SPECT-CT correlation may help to reach in proper diagnosis by avoiding misinterpretation of certain physiological or pathological conditions.

This scan may be done for the diagnosis of certain clinical conditions such as- hepatic tumors, hepatitis, cirrhosis, abscesses, cysts of the liver \& spleen. It may also be done to assess the condition of the liver or spleen after abdominal injury or when there is unexplained pain in the right upper quadrant of the abdomen. Enlargement of the liver or spleen and colloid shift may also be noted on this scan (11). When abdominalultrasound finding is inconclusive, liver-spleen scan along with SPECT-CT correlation may help to reach in proper diagnosis by avoiding misinterpretation of certain physiological or pathological conditions.

\section{CASE REPORT}

A forty years old female patient presented with anpainless abdominal lump in left upper quadrant for several years. She had no history of fever, ascites, jaundice or trauma. Liver function tests and other lab findings were unremarkable Initially, she was suspected as having a splenic mass and abdominal ultrasound revealed splenomegaly with a hypoechoic area (measures about $6 \times 3 \mathrm{~cm}$ ) in the sub-capsular region of spleensuggestive of splenic hematoma / with peri-splenic fluid collection (Figure 1). Right kidney, gall bladder and pancreas were visualized separately. 
A liver-spleen scan was asked for this patient at the scintigraphy division of NINMAS. Static images of the liver \& spleen were obtained in anterior, posterior \& lateral positions, 30 minutes after IV administration of $3.5 \mathrm{mCi}$ of $99 \mathrm{~m}-\mathrm{Tc} \mathrm{Sn}$ colloid. Transaxial, coronal \& sagittal SPECT-CT images were obtained reconstructed.

Planar images revealed hepatomegaly with elongated left lobe and normal sized spleen. There was no evidence of colloid shift (Figure 2). SPECT-CT aided the diagnosis as the most important technique and removed diagnostic dilemma.

Transaxial, coronal \& sagittal SPECT-CT images demonstrated an unusually enlarged liver occupying the part of right hypochondrium, epigastrium andlefthypochondrium. Size of the spleen was normal but markedly compressed and shifted posteriorly by the hugely enlarged left lobe of liver. Both liver and spleen concentrated radio-colloid uniformly. There was a pocket between the liver \& spleen occupied by bowel loops and no evidence of splenic hematoma (Figure: 3 ).

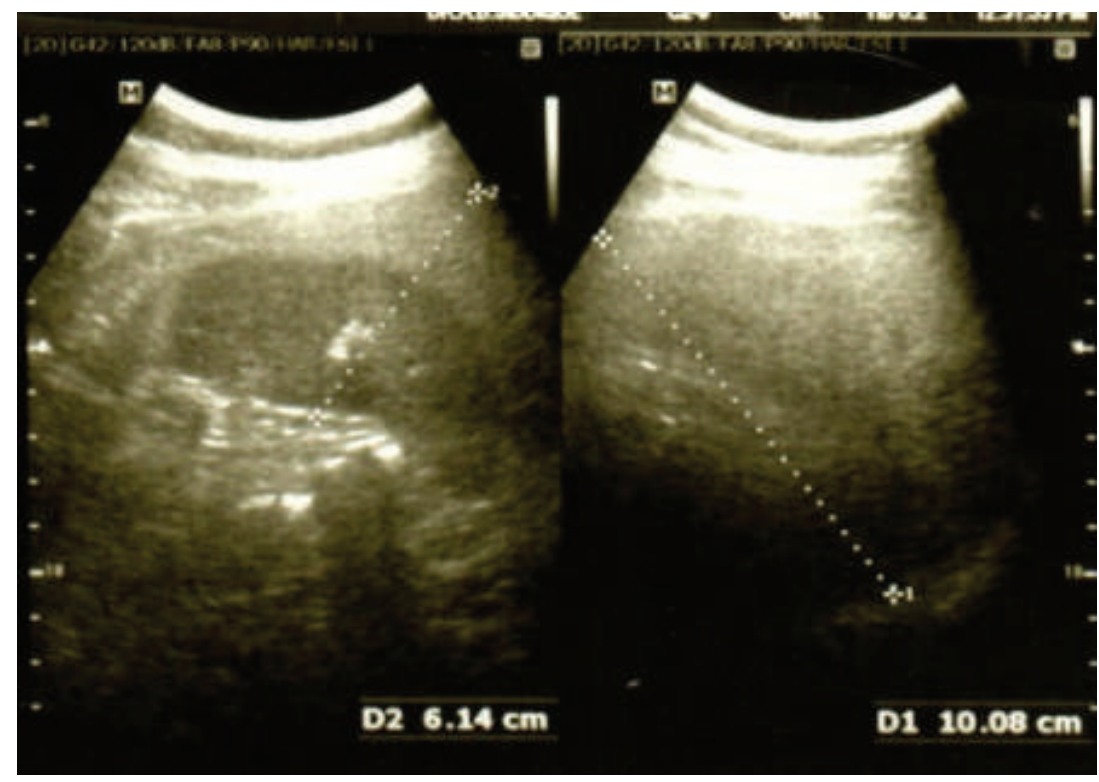

Figure 1: USG of upper abdomen revealed splenomegaly with suspected splenic hematoma.

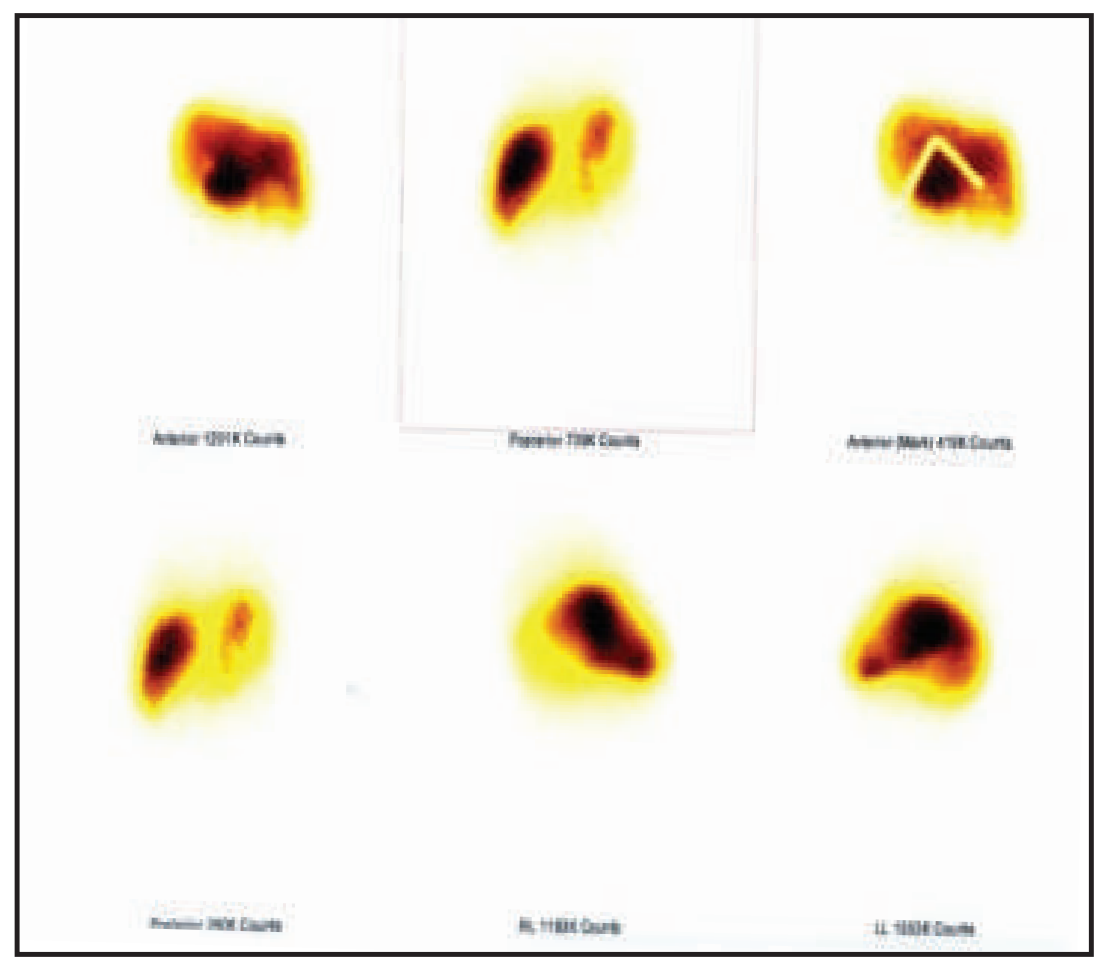

Figure 2: Planar images of liver-spleen scan showing hepatomegaly 


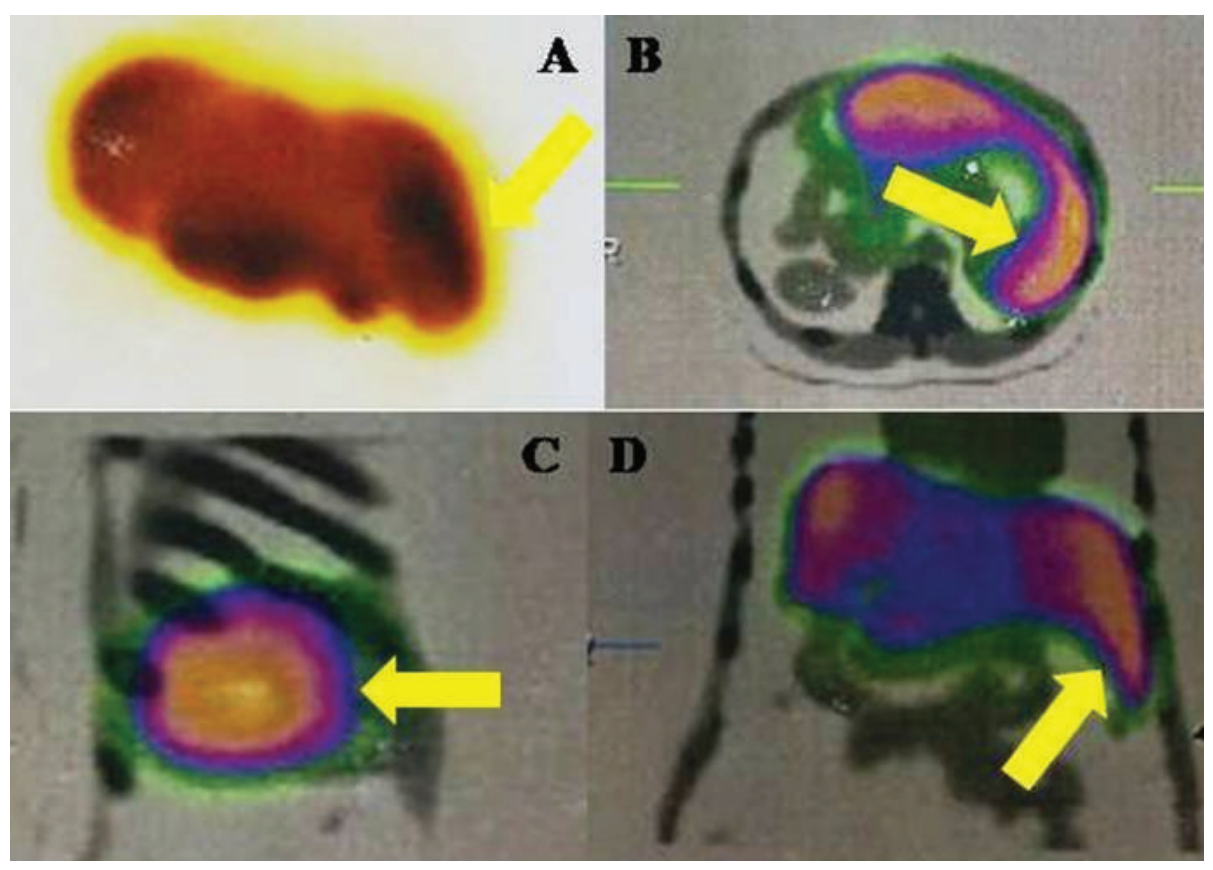

Figure 3: SPECT-CT images of liver- spleen scan of the patient with suspected splenic haematoma A. SPECT image showing liver (yellow arrow); B, C \& D: SPECT-CT image in trans-axial, sagittal and coronal sections showing unusually enlarged left lobe of liver (yellow arrow).

\section{DISCUSSION}

Liver is the largest viscera in the abdomen. It occupies right hypochondriac, small part of left hypochondriac and epigastric regions. Liver has four lobes: right lobe, left lobe, caudate lobe and quadrate lobe. The right lobe is larger than the left lobe. Embryologically, it develops from an endodermal evagination of the foregut and from the mesenchyme of the septum transversum. Hepatic diverticulum appears at four weeks of gestation and divides into right and left

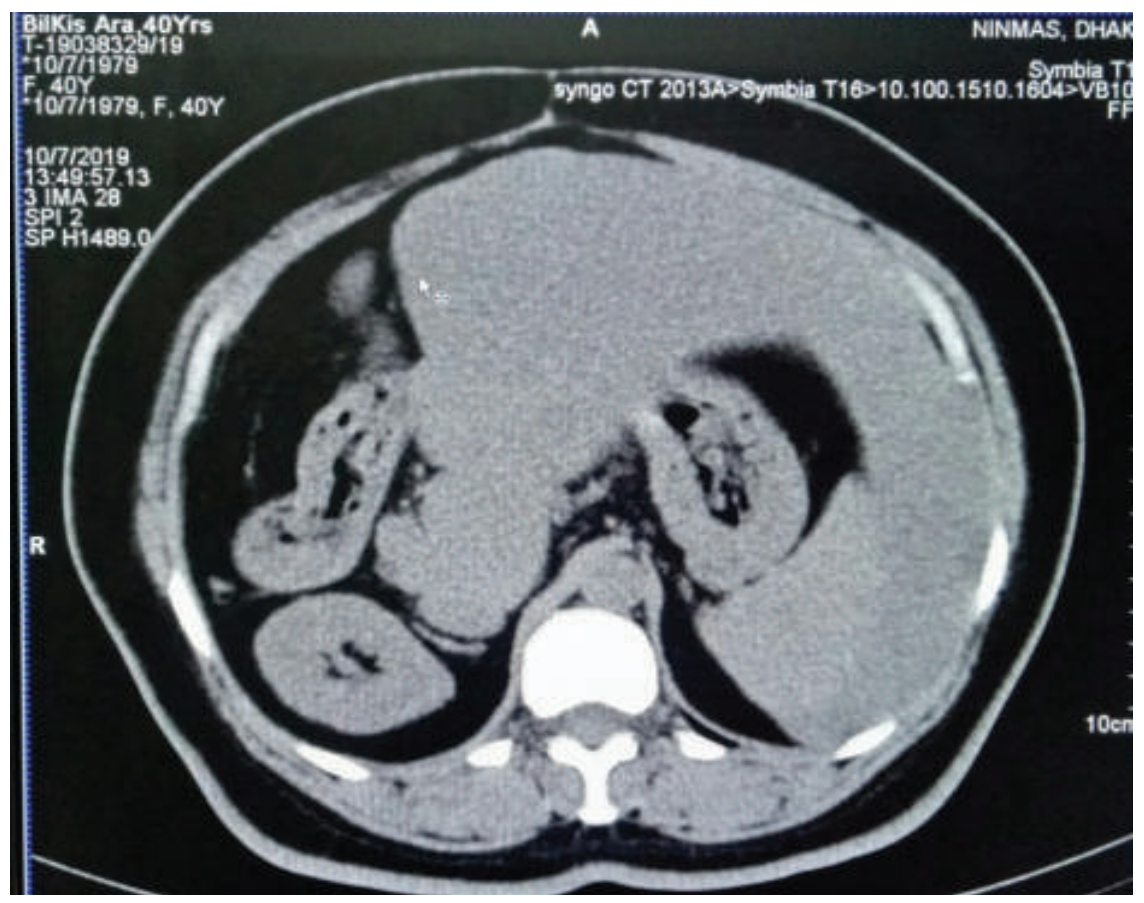

Figure 4-A: CT scan of abdomen showing an unusually enlarged left lobe of liver occupying right hypochondrium, epigastrium \& left hypochondrium regions resembling Beaver's tail. 
hepatic buds. Left lobe, caudate lobe and quadrate lobe develop from left hepatic bud and right lobe develops from right hepatic bud. At three months of gestation, the liver almost fills the abdominal cavity and its left lobe is nearly as large as its right. When haematopoietic activity of the liver is assumed by the spleen and bone marrow, the left lobe undergoes some degeneration and becomes smaller than the right $(1,2)$. Anatomical dysmorphosis is not common for liver. Morphological variations in the liver can be congenital or acquired. The congenital abnormalities of the liver include agenesis, atrophy or hypoplasia of lobes, accessory lobes, accessory fissures etc. There are few kinds of morphological variations of left lobe found in the literatures including hypertrophied/ elongated left lobe, hypoplasia of left lobe and presence of fissures. Some authors classified left lobe as a) extremely long left lobe; b) too flat like pancake; c) ligular prolongation (spatular); d) enlargement of left lobe (3).

An elongated left lobe of the liver (also known as Beaver tail liver) is defined when the left lobe of the liver extends beyond the stomach to the left and / or reaches the superior pole of the spleen on CT image.Beaver tail liver is an anatomic variation of liver where the elongated left lobe extends laterally to contact or often wrap the spleen (Figure: 4-A, B).

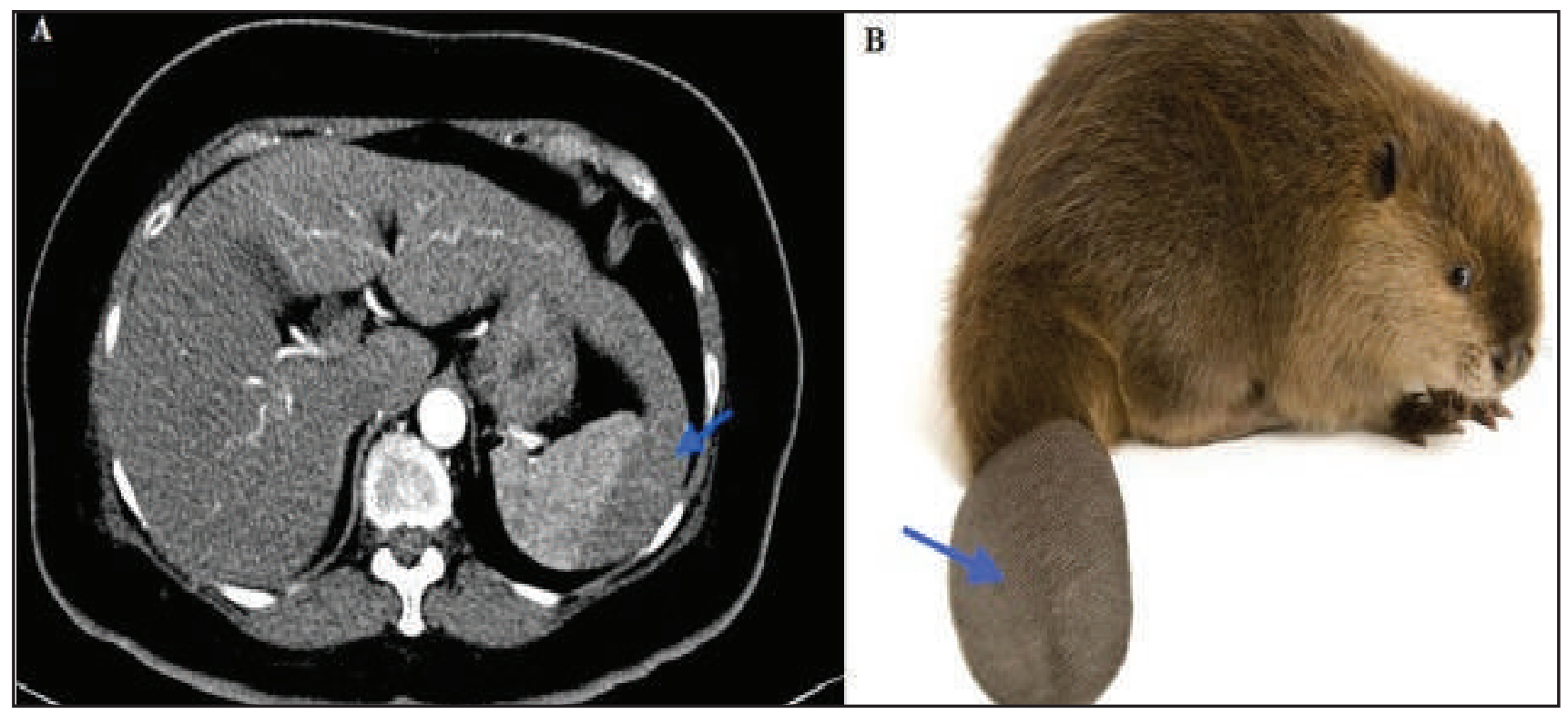

Figure 4-B: (a) CT scan of abdomen showing an unusually enlarged left lobe of liver occupying right hypochondrium, epigastrium \& left hypochondrium regions resembling Beaver's tail

(b) Beaver's tail (Acnowledgement: Faeez MS. Beaver in the liver. Pan African Medical Journal 2017 June; 27. DOI: 10.11604/pamj.2017.27.138.12227)

This variation is also termed as 'the sliver of liver'. It may be diagnosed as an incidental finding during ultrasound (4-7). Although this condition is very rare, it has some important clinical implications.

It may be misdiagnosed as distension of the splenic flexure of the colon, gastrooptosis, hydatid cyst or sarcoma of liver (3). It may also be mistaken for splenomegaly due to the fact that liver \& spleen show same echogenecity and density in ultrasonography and CT scan images and misdiagnosed as perisplenic / subcapsular hematoma or collections when they differ in density and echogenicity (4).
Perisplenic hematoma appears as 'double contour' to the spleen on ultrasonography. The initial diagnostic dielema in this case was due to double contour' sign of spleen although the patient had no history of trauma or pain in abdomen (7).Liver-spleen scintigraphy is usually able to show the area of splenic hematoma as an area of diminished uptake $(8,9)$. In this case, SPECT-CT correlated liver-spleen scintigraphy hepledto distinguish a suspected splenic hematoma or perisplenic /sub capsular collection witha hugely enlarged left lobe of liver which might be congenital. 


\section{CONCLUSION}

Left lobe megaly of liver is a rare congenital anomaly which may often mimic as splenic trauma, sub capsular or perisplenic collection or sometimes may be misdiagnosed as splenic infarction. Liver-spleen scintigraphyspecially SPECT-CT scan may be an investigation of choice in this clinical aspect. When the results are inconclusive in ultrasonogram, this nuclear medicine test can guide the physicians to avoid misinterpretations.SPECT-CT correlation have increased the specificity of this scan\& made it more popular \&reliable for the physicians regarding their diagnosis of hepatic \& splenic pathologies.

\section{REFERENCES}

1. Standring S. Gray's Anatomy. Pelvic girdle and lower limb, 40th edn. Edinburg, London, New York, Oxford, Philadelphia, St. Louis, Sydney, Toronto: Elsevier Churchill Livingstone 2008p. 1207-8.

2. Marques P, Skorupskaite K, George JT, Anderson RA. Physiology of GnRH and gonadotropin secretion. In Endotext [Internet] 2018 Jun 19. MDText. com, Inc.

3. Joshi SS, Valimbe N, Joshi SD. Morphological variations of left lobe of liver. Journal of the Anatomical Society of India. 2018 Aug 1;67:S73.
4. Jones R, Tabbut M, Gramer D. Elongated left lobe of the liver mimicking a subcapsular hematoma of the spleen on the focused assessment with sonography for trauma exam. The American journal of emergency medicine. 2014 Jul 1;32(7):814-e3.

5. Glenisson M, Salloum C, Lim C, Lacaze L, Malek A, Enriquez A, Compagnon P, Laurent A, Azoulay D. Accessory liver lobes: anatomical description and clinical implications. Journal of visceral surgery. 2014 Dec 1;151(6):451-5.

6. Wu S, Tu R, Liu G, Nan R, Guan Y, Zheng E, Zhao Y. Anatomical variation of the liver with elongated left lobe may be a trap forthe ultrasound detection of focal liver lesion. Medical Ultrasonography. 2015 Mar 1;17(1):12-5.

7. Dunlop DG, Evans RM. Congenital abnormality of the liver initially misdiagnosed as splenic haematoma. Journal of the Royal Society of Medicine. 1996 Dec;89(12):702-4.

8. Makoba IG. Splenic injury following trauma: The role of ultrasonography. African health sciences. 2001;1(1):23-5.

9. Fischer KC, Eraklis A, Rossello P, Treves S. Scintigraphy in the followup of pediatric splenic trauma treated without surgery. Journal of nuclear medicine: official publication, Society of Nuclear Medicine. 1978 Jan;19(1):3.

10. Zuckerman E, Slobodin G, Sabo E, Yeshurun D, Naschitz JE, Groshar D. Quantitative liver-spleen scan using single photon emission computerized tomography (SPECT) for assessment of hepatic function in cirrhotic patients. Journal of hepatology. 2003 Sep 1;39(3):326-32.

11. Solav SV, Patil AM, Savale SV. Radionuclide Liver-Spleen Scan to Detect Splenosis. Indian Journal of Surgery. 2019 Dec 1;81(6):602- 